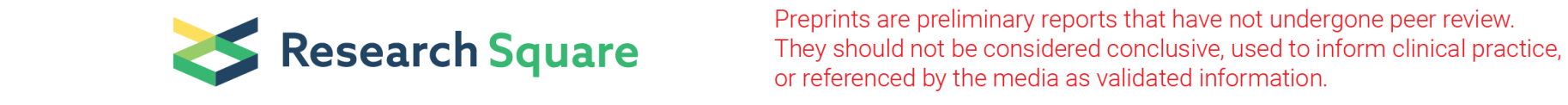

\title{
Screening and Evaluating Novel Autoantibodies Based on Proteomic Chips in Diagnosis of Esophageal Squamous Cell Carcinoma
}

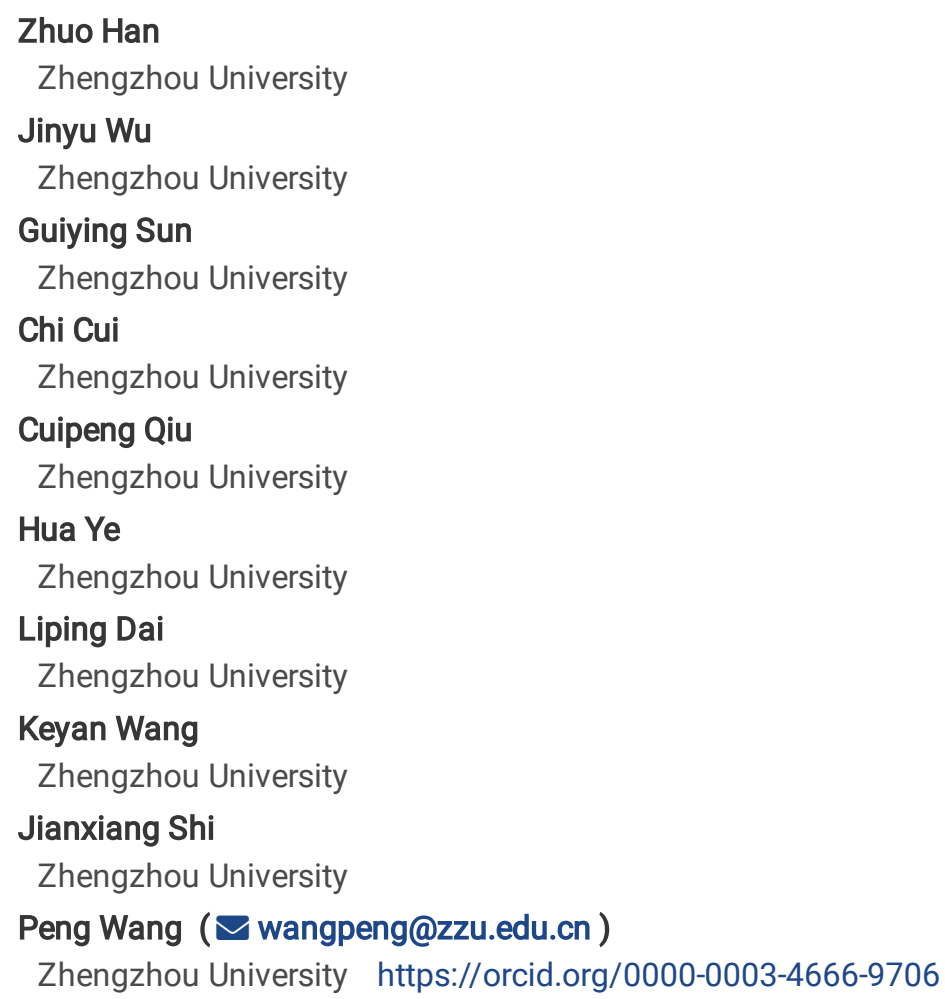

Zhuo Han

Zhengzhou University

Jinyu Wu

Zhengzhou University

Guiying Sun

Zhengzhou University

Chi Cui

Zhengzhou University

Cuipeng Qiu

Zhengzhou University

Hua Ye

Zhengzhou University

Liping Dai

Zhengzhou University

Keyan Wang

Zhengzhou University

Jianxiang Shi

Zhengzhou University

Peng Wang ( $\square$ wangpeng@zzu.edu.cn )

Zhengzhou University https://orcid.org/0000-0003-4666-9706

\section{Primary research}

Keywords: Esophageal squamous cell carcinoma (ESCC), proteomic chips, tumor-associated antigen (TAA), autoantibodies, diagnostic model.

Posted Date: November 30th, 2021

DOI: https://doi.org/10.21203/rs.3.rs-1085112/v1

License: @ (i) This work is licensed under a Creative Commons Attribution 4.0 International License. Read Full License 


\section{Abstract \\ Background}

More and more studies have confirmed that TAAbs could be used as potential biomarkers for tumor patients. The aim of this study is to identify novel TAAbs through proteomic chips and construct a diagnostic model to discriminate esophageal squamous cell carcinoma (ESCC) cases from benign esophageal diseases cases and normal controls (NCs).

\section{Methods}

The human proteomic chips were used to screen TAAbs. Enzyme-linked immunosorbent assay (ELISA) was adopted to verify and validate the candidate TAAbs which were screened by the chips in verification phase ( 90 ESCC cases and 90 NCs) and validation phase (126 ESCC cases, 237 benign esophageal diseases cases and 126 NCs). Based on the candidate TAAbs, then the diagnostic model for ESCC was constructed by logistic regression analysis in the training group and validated in the testing group.

\section{Results}

Firstly, thirteen potential candidate TAAbs were identified by proteomic chips. In verification phase, the titers of six TAAbs (antiMAGEA1, anti-VCL, anti-PRKCZ, anti-TP53, anti-NFKB1 and anti- MAGEA4) in ESCC cases were higher than those in NCs while other seven TAAbs showed no difference. Subsequently, six candidate TAAbs were validated further in validation phase. Finally, the logistic regression model with 3 TAAbs (anti-MAGEA1, anti-VCL, anti-TP53) could discriminate ESCC cases from NCs with area under curve(AUC) of 0.80 in the training group and 0.73 in the testing group, respectively. Meanwhile, the model could discriminate ESCC cases from benign esophageal diseases cases with AUC of 0.74 .

\section{Conclusion}

The study has identified six novel TAAbs through protein chips and constructed a diagnostic model. The panel showed great performance to distinguish ESCC cases from benign esophageal diseases cases and NCs.

\section{Background}

Esophageal cancer囚ECXis one of the common malignant tumors in the world, and its incidence and mortality are relatively high. Global cancer statistics released in 2020 showed that EC has a higher incidence in East Asia, especially in China, and the incidence in males are significantly higher than that in females[1]. In 2020, the number of new cases and deaths of EC in China are 324,422 and 301,135, accounting for about $50 \%$ and $55 \%$ of the world[1]. According to the clinical histopathological type, EC was mainly divided into esophageal adenocarcinoma (EAC) and esophageal squamous cell carcinoma (ESCC) [1].Importantly, the incidence of ESCC in China accounts for more than $70 \%$ of the world[2]. The onset of ESCC was insidious and there were no obvious clinical symptoms in the early stage[3]. At present, the pathogenesis of ESCC was not clear, and there were few effective treatment measures for advanced ESCC patients. Therefore, seeking a noninvasive diagnostic method for ESCC of early stage is crucial to improve the 5-year survival rate of patients. At present, the clinical diagnosis of ESCC was mainly based on clinical symptoms combined with cytological examination, endoscopy, other imaging and pathological cell techniques[4]. Due to the disadvantages which still existed such as low sensitivity, high cost and invasiveness, they were not suitable to use as screening tests for ESCC.So far, studies have found kinds of molecular biomarkers related to the diagnosis of ESCC, including cancer stem cells (CSCs) and serological markers CEA, CA199 and SCC etc., while there was still no consensus that a certain biomarker can be used in clinical practice[5, 6].Therefore, actively exploring novel biomarkers for diagnosis of ESCC has important clinical significance for improving the prognosis of patients.

Previous studies have demonstrated that tumor-associated antigens (TAAs) can trigger immune response to produce autoantibodies against TAAs (TAAbs) during the process of tumor growth[7, 8]. In recent years, more and more studies have confirmed that TAAbs could be used as potential biomarkers for tumor patients[9-14]

Protein chip technology is playing an increasingly important role in the study of screening autoantibodies against tumor-associated antigens owing to its advantages of high-throughput, parallel and rapid analysis[15-19]. This study devoted to screen and validate 
novel TAAbs related to ESCC through the proteomic chips (containing more than 21,000 recombinant human proteins) and Enzymelinked immunosorbent assay (ELISA), then a diagnostic model was constructed for evaluating the diagnostic value in ESCC.

\section{Methods}

\subsection{Serum sample}

Serum samples of ESCC cases and benign esophageal diseases (BED) cases were collected from Affiliated Tumor Hospital and the First Affiliated Hospital of Zhengzhou University (June 2013-December 2017). All enrolled ESCC cases were confirmed by pathological examination and did not receive any treatment. The benign esophageal diseases (BED) cases have no tumor-related diseases. The NCs came from the biological sample bank of the Key Laboratory of Tumor Epidemiology of Henan Province and did not have any digestive tract diseases or autoimmune diseases. This study was designed in three phases. In the discovery phase, 30 ESCC sera in 10 sera pools (three serum samples were mixed into one sera pool) and $21 \mathrm{NCs}$ sera in 7 sera pools (three serum samples were mixed into one sera pool) and 3 individual sera were used to screen candidate TAAbs. In the verification phase and validation phase, a total of 669 serum samples were used to verify and validate candidate TAAbs through Enzyme-linked immunosorbent assay (ELISA), including 216 ESCC cases, 237 benign esophageal diseases (BED) cases and 216 normal controls (NCs). The verification phase included 90 ESCC cases and 90 NCs. The validation phase included 126 ESCC cases, 126 NCs and 237benign esophageal diseases (BED) cases. All ESCC cases and NCs have no difference between age and genderafter frequency matching. All serum samples were collected by standard methods and immediately taken to the laboratory for storage. This study has been approved by the Ethics Committee of Zhengzhou University and all participants have informed consent forms.

\subsection{Proteomic chips}

In this study, a small number of serum samples (10 ESCC serum pools vs $10 \mathrm{NC}$ serum pools) were used for screening TAAbs through the HuProt ${ }^{\mathrm{TM}}$ human proteomic chips.HuProt ${ }^{\mathrm{TM}}$ human proteomic chips were purchased from BCBIO Biotechnology (Guangzhou, China).The chips are currently the largest collection of full-length human proteins available in the world. It contains 20,240 recombinant proteins, covering $81 \%$ of the human proteome. More detailed information about proteomic chips was available at https://cdi. bio/huprot/.

\subsection{Enzyme-linked immunosorbent assay}

Enzyme-linked immunosorbent assay (ELISA) was applied to detect the level of TAAbs in serum samples. The protocol has been described in detail in our previous studies[20,21]. In this study, the verification phase was used to verify the candidate TAAbs that screened out from the proteomic chips, then the TAAbswere validated in the validation phase. The 13 recombinant proteins were diluted in coating buffer at optimal concentrations of $0.25,0.25,0.25,0.25,0.25,0.25,0.25,0.25,0.125,0.125,0.25,0.25 \mathrm{and} 0.125 \mathrm{ug} / \mathrm{ml}$ (MAGEA1, VCL, PPP1R15A, PDE4DIP, PRKCZ, TP53, SMARCC1, BASP1, SMARCE1, GABPA, ANKHD1, NFKB1, MAGEA4), respectively. Importantly, 6 same repeated serum samples and 2 blank controls were run on each plate. The mean value of the 8 samples was used to normalize the difference plates,and the blank controls were consulted for quality control for individual plates.

\subsection{Statistical analysis}

The human proteomic chips were used to screen TAAbs, and the detected data were normalized based on signal-to-noise ratio (SNR) value. Whereafter,the SNR valuesof each sample were analyzed by the z-score method, and then the differentially expressed TAAbs were found out. The FunRich website was applied to find proteins related to cancer pathways (GO, KEGG pathways), focusing on cell cycle regulation, apoptosis, signal transduction and expression locations. SPSS 26.0, GraphPad Prism 6.0 and MedCalc 11.0 were used to analyze the experimental data. PASS 15.0 software was used to estimate the sample size. Non-parametric tests were adopted to analyze whether there were differences between groups; ROC curve was used to evaluate the diagnostic value of a single TAAb. The sensitivity, specificity, accuracy, positive predictive value, negative predictive value, positive likelihood ratio, negative likelihood ratio and Youden index were calculated according to ROC curve (the cut-off value of single TAAb was determined according to the maximum Youden index and the specificity was greater than $90 \%$ ). Logistic regression was used to construct the diagnosis model of ESCC. All $P$ values were based on two-sided test, and $P<0.05$ was considered to be statistically different.

\section{Results}




\subsection{Baseline characteristics of the study population}

The PASS software was used to calculate the sample size. The parameter was set as follows: $a=0.05 ; \delta=0.05-0.1$; sensitivity $=20 \%$; specificity $=90 \%$, then the range of sample size was $70-264$. This study was designed in three phases. The first phase was the discovery phase, including $10 \mathrm{ESCC}$ sera pools and $10 \mathrm{NCs}$ sera pools, the high-throughput protein chips were used to screen candidate TAAbs related to the diagnosis of ESCC. The second phase was the verification phase, including 90 ESCC cases and 90 NCs, ELISA was used to verify the 13 TAAbs screened by the protein chips. The third phase was the validation phase, including 126 ESCC cases, 237 benign esophageal diseases (BED) cases and 126 NCs. Furthermore, the candidate TAAbs screened out were validated by ELISA. The design of the present study was shown in Figure 1. The clinical baseline characteristics of the subjects in our studies were listed in Table 1. The sample size of verification phase and validation phase were within the range. And there was no significant difference in age and gender between the verification phase and validation phase.

\subsection{The process of screening TAAbs}

Firstly, the data of chips was used to screen the TAAbs related to the cancer pathway (GO, KEGG pathway) through the FunRich website with focusing on cell cycle regulation, apoptosis and signal transduction, expression position. The figure \$1 presented the GO and KEGG pathway. A total of 25 TAAbs were screened out. Secondly, the fold change $\geq 2$ and histogram were adopted to observe the TAAs with significant differences as a further step to screen the candidate TAAs. Subsequently, searched protein function and related literature through the Uniprot website and Pubmed. Finally, 13 TAAbs (anti-PDE4DIP, anti-VCL, anti-TP53, anti-PPP1R15A, antiSMARCC1, anti-BASP1, anti-SMARCE1, anti-GABPA, anti-NFKB1, anti-MAGEA4, anti-PRKCZ, anti-MAGEA1, anti-ANKHD1) were screened out. In addition, the mRNA expression level of 13 proteins of ESCA in GEPIA (|Log2FC| Cutoff $=0.485$, $p$-value Cutoff $=0.01$ ) were shown in Figure $\mathbf{S 2}$.

\subsection{Autoantibodies in the verification phase and the validation phase}

To verify the 13 TAAbs screened out by the chips, the ELISA was carried out to verify and validate in the verification phase and the validation phase, respectively. The verification phase included 90 ESCC cases and 90 NCs. Figure 2A showed the optical density (OD) value of single TAAb in the verification phase. It indicated that the titers of six autoantibodies (anti-MAGEA1, anti-NFKB1, anti-VCL, antiMAGEA4, anti-PRKCZ and anti-TP53) in sera were significantly higher than normal controls. However, the titers of anti-PPP1R15A, antiSMARCC1, anti-SMARCE1, anti-GABPA, anti-PDE4DIP and anti-BASP1 in sera did not show significant differences compared with normal controls. Figure 3 showed the diagnostic value of the single TAAb. The range of AUC was $0.51-0.68$, the range of sensitivity was from $1.1 \%$ to $35.56 \%$, and all of the specificity were greater than $90.0 \%$.

The validation phase included 126 ESCC cases, 126 NCs and 237 benign esophageal diseases (BED) cases. Figure 2B showed the OD value of the six TAAbs screened out by the verification phase. It indicated that the titers of six autoantibodies (anti-MAGEA1, antiNFKB1, anti-VCL, anti-MAGEA4, anti-PRKCZ and anti-TP53) in the sera of patients with ESCC were also significantly higher than thatin benign esophageal diseases (BED) cases and normal controls. Figure 4 showed the diagnostic value of the six autoantibodies screened out by the verification phase. The range of AUC was $0.63-0.74$, the sensitivity was from $32.5 \%$ to $40.5 \%$, and the specificity were greater than $90.0 \%$. Anti-MAGEA1 presented an outstanding diagnostic value, with an AUC of 0.74 , sensitivity of $40.48 \%$, specificity of $90.11 \%$ among six TAAbs in the validation phase. Anti-TP53 achieved the favorable specificity of $91.45 \%$. The diagnostic value of single TAAb in verification phase and validation phase that discriminate ESCC cases from NCs were shown in Table 2.Furthermore, the diagnostic value of single TAAb that discriminates ESCC cases from benign esophageal diseases (BED) cases were shown in Table 3. The range of AUC was $0.65-0.76$, the sensitivity was from $32.54 \%$ to $43.65 \%$, and the specificity were greater than 90.0\%. However, the Figure S3 showed that only anti-MAGEA1 could discriminate benign esophageal diseases (BED) cases from NCs among six TAAbs, the AUC was 0.591(95\%Cl:0.529,0.652).

\subsection{Establishment of diagnostic model to discriminate ESCC from NC or BED}

Validation phase served as a training group to construct diagnostic model for ESCC. The dependence variable was whether a participant was considered as ESCC or not. Six TAAbs with different titers between ESCC cases and NCs were used as independence variables. The Logistic regression model was produced with three TAAbs. The formula of the prediction model was $\operatorname{PRE}(P=E S C C, 3 T A A b s)=1 /(1+\operatorname{EXP}(-(-4.810+5.213 \times$ MAGEA1 $+4.699 \times \mathrm{VCL}+6.190 \times \mathrm{TP} 53)))$. In the training group, the AUC was 0.80 , the sensitivity and the specificity were $61.1 \%, 90.1 \%$, respectively (Figure $5 \mathrm{~A}$ ). Verification phase was used to test the model. The AUC of the testing group was 0.73 , the sensitivity and the specificity were $65.6 \%, 72.2 \%$, respectively (Figure $5 \mathrm{~B}$ ). The model was stable 
by the Delong test $(P=0.160)$. Furthermore, the model had great performance to distinguish ESCC cases and benign esophageal diseases (BED) cases, with the AUC of 0.75 , the sensitivity of $78.6 \%$ and the specificity of $59.0 \%$ (Figure 5 C).

\subsection{Subgroup analysis of diagnostic model}

Patients with ESCC were divided into different subgroups according to clinical characteristics (Table 4). The results indicated that the diagnostic modeldid not perform well among the subgroups of clinical characteristics of ESCC (age, gender, family history, differentiated degree, TNM stage, degree of invasion). And the validation phase also showed similar results.

\section{Discussion}

According to the released global cancer data, the incidence of EC ranked seventh, and the mortality rate ranked sixth. East Asia had the highest incidence of EC, and China had the heaviest burden[1]. The current clinical treatment of EC included surgery, targeted drug therapy, adjuvant radiotherapy and chemotherapy[22-27]. However, only relying on current medical technology, the five-year survival rate was only between $15 \%-20 \%{ }^{[3]}$. If EC can be detected at early stage, the 5 -year survival rate could be increased by $80 \%-90 \%[4]$. Therefore, improving the screening and diagnosis ability of EC was the most economical and effective means of prevention and treatment. Current diagnostic approaches may have various shortcomings in clinical diagnosis of EC, such as low sensitivity and traumatic[28-30]. The appearance of autoantibodies against tumor-associated antigens can make up these shortcomings and provide an opportunity for auxiliary diagnosis of esophageal cancer[9, 31-33].

In this study, thirteen tumor-associated antigens related to the diagnosis of EC were screened out through the protein chips. Then the corresponding thirteen autoantibodies were screened further through ELISA to confirm whether they have diagnostic value for ESCC. Finally, we constructed the logistic regression model for diagnosing ESCC, including anti-MAGEA1, anti-VCL and anti-TP53 in the model. The AUC of the diagnostic model in the training group was 0.80 , and the sensitivity and specificity were $60.1 \%$ and $90.1 \%$, respectively. The model was found to be stable by the Delong test. The results of this study confirmed the TAAbs were reliable as biomarkers for the diagnosis of ESCC.

Many researchers have performed similar studies. In 2014, Xu discovered a panel of autoantibodies against tumor-associated antigens (TP53, NY-ESO-1, MMP-7, HSP70, peroxiredoxin VI and Bmi-1) for ESCC with AUC of 0.78[34, 35].In 2018, Zhang found that antiTOPO48 could be used as a biomarker for early diagnosis and prognosis of ESCC, the area under the ROC curve was 0.863[36]. Our study produced consistent results that anti-TP53 could be a biomarker for detection of ESCC, meanwhile, the study also found other five potential TAAbs (anti-MAGEA1, anti-NFKB1, anti-VCL, anti-MAGEA4, anti-PRKCZ). A study found that a panel of three TAAbs (antiHCCR, anti-C-myc and anti-MDM2) and three miRNAs (miR-21, miR-223 and miR-375) had a high diagnostic value for ESCC with AUC of 0.89 , while too many biomarkers combined in the study may not be conducive to large-scale screening and cause more economic burden for patients[37]. Therefore, the author also discovered three TAAbs (anti-GNA11, anti-ACVR1B and anti-TP53) and constructed a model that the diagnostic value of ESCC was 0.88 [38]. A protein chips customized by driver gene were used in the study, however, our study screen novel biomarkers through the comprehensive protein chips. Previous studies have indicated that the sensitivity of single TAAb was usually low, our study also adopted the approach of several combined TAAb(s to improve the sensitivity, specificity and diagnostic value. Furthermore, our study also showed the TAAbs have the ability to differentiate ESCC cases from benign esophageal diseases (BED) cases.

As far as we know, the panel of the three TAAbs in the current study have not been studied in the diagnosis of ESCC. MAGEA1 mainly existed in cells and could play a role in embryonic development and tumor transformation or tumor progression. The related studies have showed that it was highly expressed in endometrial cancer, lung cancer and other cancers, and it may be also a prognostic factor in ESCC[39-41].VCL mainly existed on the cell membrane and was mainly involved in cell adhesion. It was highly expressed in pancreatic cancer tissue and was related to the poor prognosis of cancer [33, 42].TP53 was mainly present in the cytoplasm and nucleus, inducing growth arrest or apoptosis, regulating cell cycle. It was highly expressed in breast cancer, ovarian cancer, esophageal cancer, colorectal cancer tissues and cells and its autoantibodies were also found to be highly expressed in cancer[43-47].

The advantage of our studies lies in the screening of tumor-associated antigens related to the diagnosis of ESCC through the human proteomic chips. In addition, we constructed a diagnostic model combining threeTAAbs to improve the efficiency of ESCC diagnosis and verified the stability of the model. Of course, this study is not flawless. More TAAbs have been screened out through the protein chips. This study may only verify a small number of them, and there are more potential markers waiting for our verification. 


\section{Conclusion}

In conclusion, this study has identified six TAAbs biomarkers as an auxiliary diagnostic method for ESCC and showed the diagnostic model constructed by the combination of three TAAbs, which can distinguish ESCC cases from NCs and benign esophageal diseases (BED) cases. Furthermore, the related mechanism of these TAAbs in ESCC is also worthy of our further exploration.

\section{Abbreviations}

ESCC: esophageal squamous cell carcinoma

NCs: normal controls

ELISA: enzyme-linked immunosorbent assay

TAAbs: autoantibodies against the tumor-associated antigens

TAA: tumor-associated antigen

AUC: area under curve

EC:esophageal cancer

EAC: esophageal adenocarcinoma

CSCs: cancer stem cells

BED: benign esophageal diseases

OD: optical density

\section{Declarations}

\section{Ethics approval and consent to participate}

Informed consent forms have been signed by all participates before this study. The research protocol has been approved by the Ethics Committee of the Zhengzhou University and is based on the ethical principles of medical research involving human subjects in the Helsinki Declaration.

\section{Consent for publication}

Not applicable.

\section{Availability of data and materials}

The datasets used during the current study are available from the corresponding author on reasonable request.

\section{Competing interests}

All authors declared that they have no conflict of interests.

\section{Funding}

This study supported by the Project of International Training of High-level talents in Henan Province, and Zhengzhou Major Project for Collaborative Innovation(18XTZX12007).

\section{Authors' contributions}

Zhuo Han, Guiying Sun and Peng Wang was responsible for study conception and design; Jinyu Wu and Zhuo Han were responsible forexperiment; Zhuo Han was responsible for data analysis, Keyan Wang, CuipengQiu, Chi Cui, Hua Ye, Liping Dai, Jianxiang Shi, and 
Jianying Zhang were responsible for revision of the manuscript.

Acknowledgements

No Application.

\section{References}

1. Sung H, Ferlay J, Siegel RL, Laversanne M, Soerjomataram I, Jemal A, Bray F: Global cancer statistics 2020: GLOBOCAN estimates of incidence and mortality worldwide for 36 cancers in 185 countries. CA: a cancer journal for clinicians 2021.

2. Wang Q, Liu H, Liu Z, Yang L, Zhou J, Cao X, Sun H: Circ-SLC7A5, a potential prognostic circulating biomarker for detection of ESCC. Cancer genetics 2020, 240:33-39.

3. Talukdar FR, di Pietro M, Secrier M, Moehler M, Goepfert K, Lima SSC, Pinto LFR, Hendricks D, Parker MI, Herceg Z: Molecular landscape of esophageal cancer: implications for early detection and personalized therapy. Annals of the New York Academy of Sciences 2018, 1434(1):342-359.

4. Codipilly DC, Qin Y, Dawsey SM, Kisiel J, Topazian M, Ahlquist D, lyer PG: Screening for esophageal squamous cell carcinoma: recent advances. Gastrointestinal endoscopy 2018, 88(3):413-426.

5. Chu LY, Peng YH, Weng XF, Xie JJ, Xu YW: Blood-based biomarkers for early detection of esophageal squamous cell carcinoma. World journal of gastroenterology 2020, 26(15):1708-1725.

6. Liu K, Zhao T, Wang J, Chen Y, Zhang R, Lan X, Que J: Etiology, cancer stem cells and potential diagnostic biomarkers for esophageal cancer. Cancer letters 2019, 458:21-28.

7. Tan EM, Zhang J: Autoantibodies to tumor-associated antigens: reporters from the immune system. Immunological reviews 2008, 222:328-340.

8. Tan EM: Autoantibodies, autoimmune disease, and the birth of immune diagnostics. The Journal of clinical investigation 2012, 122(11):3835-3836.

9. Zhang JY, Tan EM: Autoantibodies to tumor-associated antigens as diagnostic biomarkers in hepatocellular carcinoma and other solid tumors. Expert review of molecular diagnostics 2010, 10(3):321-328.

10. Koziol JA, Imai H, Dai L, Zhang JY, Tan EM: Early detection of hepatocellular carcinoma using autoantibody profiles from a panel of tumor-associated antigens. Cancer immunology, immunotherapy : C/l 2018, 67(5):835-841.

11. Evdokimova VN, Butterfield LH: Alpha-fetoprotein and other tumour-associated antigens for immunotherapy of hepatocellular cancer. Expert opinion on biological therapy 2008, 8(3):325-336.

12. Ibrahim AN, Robinson RA, Marr L, Abdelal AT, Nahmias AJ: Tumor-associated antigens in cervical cancer tissues and in sera from patients with cervical cancer or with head and neck cancer. Journal of the National Cancer Institute 1979, 63(2):319-323.

13. Luna Coronell JA, Syed P, Sergelen K, Gyurján I, Weinhäusel A: The current status of cancer biomarker research using tumourassociated antigens for minimal invasive and early cancer diagnostics. Journal of proteomics 2012, 76 Spec No.:102-115.

14. Shi JX, Qin JJ, Ye H, Wang P, Wang KJ, Zhang JY: Tumor associated antigens or anti-TAA autoantibodies as biomarkers in the diagnosis of ovarian cancer: a systematic review with meta-analysis. Expert review of molecular diagnostics 2015, 15(6):829-852.

15. Ma Y, Wang X, Qiu C, Qin J, Wang K, Sun G, Jiang D, Li J, Wang L, Shi J et al: Using protein microarray to identify and evaluate autoantibodies to tumor-associated antigens in ovarian cancer. Cancer science 2021, 112(2):537-549.

16. Cui C, Duan Y, Qiu C, Wang P, Sun G, Ye H, Dai L, Han Z, Song C, Wang K et al: Identification of Novel Autoantibodies Based on the Human Proteomic Chips and Evaluation of Their Performance in the Detection of Gastric Cancer. Frontiers in oncology 2021, 11:637871.

17. Liao W, Guo S, Zhao XS: Novel probes for protein chip applications. Frontiers in bioscience : a journal and virtual library 2006, 11:186-197.

18. Vlahou A, Schellhammer PF, Wright GL, Jr.: Application of a novel protein chip mass spectrometry technology for the identification of bladder cancer-associated biomarkers. Advances in experimental medicine and biology 2003, 539(Pt A):47-60.

19. Zhu H, Snyder M: Protein chip technology. Current opinion in chemical biology 2003, 7(1):55-63.

20. Wang S, Qin J, Ye H, Wang K, Shi J, Ma Y, Duan Y, Song C, Wang X, Dai L et al: Using a panel of multiple tumor-associated antigens to enhance autoantibody detection for immunodiagnosis of gastric cancer. Oncoimmunology 2018, 7(8):e1452582. 
21. Ren $P$, Ye H, Dai L, Liu M, Liu X, Chai Y, Shao Q, Li Y, Lei N, Peng B et al: Peroxiredoxin 1 is a tumor-associated antigen in esophageal squamous cell carcinoma. Oncology reports 2013, 30(5):2297-2303.

22. Egyud MR, Tseng JF, Suzuki K: Multidisciplinary Therapy of Esophageal Cancer. The Surgical clinics of North America 2019, 99(3):419-437.

23. Hirano H, Kato K: Systemic treatment of advanced esophageal squamous cell carcinoma: chemotherapy, molecular-targeting therapy and immunotherapy. Japanese journal of clinical oncology 2019, 49(5):412-420.

24. Leng XF, Daiko H, Han YT, Mao YS: Optimal preoperative neoadjuvant therapy for resectable locally advanced esophageal squamous cell carcinoma. Annals of the New York Academy of Sciences 2020, 1482(1):213-224.

25. Ma S, Paiboonrungruan C, Yan T, Williams KP, Major MB, Chen XL: Targeted therapy of esophageal squamous cell carcinoma: the NRF2 signaling pathway as target. Annals of the New York Academy of Sciences 2018, 1434(1):164-172.

26. Tsou YK, Lee CH, Le PH, Chen BH: Adjuvant therapy for pT1a-m3/pT1b esophageal squamous cell carcinoma after endoscopic resection: Esophagectomy or chemoradiotherapy? A critical review. Critical reviews in oncology/hematology 2020, 147:102883

27. Lee SL, Yadav P, Starekova J, Christensen L, Chandereng T, Chappell R, Reeder SB, Bassetti MF: Diagnostic Performance of MRI for Esophageal Carcinoma: A Systematic Review and Meta-Analysis. Radiology 2021:202857.

28. Pan J, Zheng QZ, Li Y, Yu LL, Wu QW, Zheng JY, Pan XJ, Xie BS, Wu YA, Qian J et al: Discovery and Validation of a Serologic Autoantibody Panel for Early Diagnosis of Esophageal Squamous Cell Carcinoma. Cancer epidemiology, biomarkers \& prevention : a publication of the American Association for Cancer Research, cosponsored by the American Society of Preventive Oncology 2019, 28(9):1454-1460.

29. Shen Y, Ding Y, Ma Q, Zhao L, Guo X, Shao Y, Niu C, He Y, Zhang F, Zheng D et al: Identification of Novel Circulating miRNA Biomarkers for the Diagnosis of Esophageal Squamous Cell Carcinoma and Squamous Dysplasia. Cancer epidemiology, biomarkers \& prevention : a publication of the American Association for Cancer Research, cosponsored by the American Society of Preventive Oncology 2019, 28(7):1212-1220.

30. Xie B, Lin J, Sui K, Huang Z, Chen Z, Hang W: Differential diagnosis of multielements in cancerous and non-cancerous esophageal tissues. Talanta 2019, 196:585-591.

31. Gao HJ, Yue ZG, Zheng M, Zheng ZX: Identification and expression of a tumor-associated antigen in esophageal squamous cell carcinoma. Zhongguo yi xue ke xue yuan xue bao Acta Academiae Medicinae Sinicae 2012, 34(3):244-248.

32. Ren S, Zhang S, Jiang T, He Y, Ma Z, Cai H, Xu X, Li Y, Cai W, Zhou J et al: Early detection of lung cancer by using an autoantibody panel in Chinese population. Oncoimmunology 2018, 7(2):e1384108.

33. Zhou Y, Cui J, Du H: Autoantibody-targeted TAAs in pancreatic cancer: A comprehensive analysis. Pancreatology : official journal of the International Association of Pancreatology (IAP) [et al] 2019, 19(5):760-768.

34. Xu YW, Peng YH, Chen B, Wu ZY, Wu JY, Shen JH, Zheng CP, Wang SH, Guo HP, Li EM et al: Autoantibodies as potential biomarkers for the early detection of esophageal squamous cell carcinoma. The American journal of gastroenterology 2014, 109(1):36-45.

35. Xu YW, Chen H, Guo HP, Yang SH, Luo YH, Liu CT, Huang XY, Tang XM, Hong CQ, Li EM et al: Combined detection of serum autoantibodies as diagnostic biomarkers in esophagogastric junction adenocarcinoma. Gastric cancer : official journal of the International Gastric Cancer Association and the Japanese Gastric Cancer Association 2019, 22(3):546-557.

36. Zhang JB, Cao M, Chen J, Ye SR, Xie K, He X, Ma XL, Zhang J, Yie SM: Serum anti-TOPO48 autoantibody as a biomarker for early diagnosis and prognosis in patients with esophageal squamous cell carcinoma. Clinics and research in hepatology and gastroenterology 2018, 42(3):276-284.

37. Sun G, Ye H, Wang X, Li T, Jiang D, Qiu C, Dai L, Shi J, Wang K, Song C et al: Autoantibodies against tumor-associated antigens combined with microRNAs in detecting esophageal squamous cell carcinoma. Cancer medicine 2020, 9(3):1173-1182.

38. Sun G, Ye H, Wang X, Cheng L, Ren P, Shi J, Dai L, Wang P, Zhang J: Identification of novel autoantibodies based on the protein chip encoded by cancer-driving genes in detection of esophageal squamous cell carcinoma. Oncoimmunology 2020, 9(1):1814515.

39. Schooten E, Di Maggio A, van Bergen En Henegouwen PMP, Kijanka MM: MAGE-A antigens as targets for cancer immunotherapy. Cancer treatment reviews 2018, 67:54-62.

40. Srdelić S, Kuzmić-Prusac I, Spagnoli GC, Juretić A, Čapkun V: MAGE-A4 and MAGE-A1 Immunohistochemical Expression in Highgrade Endometrial Cancer. International journal of gynecological pathology : official journal of the International Society of Gynecological Pathologists 2019, 38(1):59-65. 
41. Yu Y, Huang C, Li Z, Zhao F, Zhou Y, Li J, Zhu C, Li Q, Zhuang Y, Xu J et al: Expressions of melanoma-associated antigen A1 as a prognostic factor in Chinese patients with resectable oesophageal squamous cell carcinoma. Interactive cardiovascular and thoracic surgery 2019, 29(4):510-516.

42. Liu S, Wang W, Zhao Y, Liang K, Huang Y: Identification of Potential Key Genes for Pathogenesis and Prognosis in Prostate Cancer by Integrated Analysis of Gene Expression Profiles and the Cancer Genome Atlas. Frontiers in oncology 2020, 10:809.

43. Petitjean A, Achatz MI, Borresen-Dale AL, Hainaut P, Olivier M: TP53 mutations in human cancers: functional selection and impact on cancer prognosis and outcomes. Oncogene 2007, 26(15):2157-2165.

44. Silwal-Pandit L, Langerød A, Børresen-Dale AL: TP53 Mutations in Breast and Ovarian Cancer. Cold Spring Harbor perspectives in medicine 2017, 7(1).

45. Schon K, Tischkowitz M: Clinical implications of germline mutations in breast cancer: TP53. Breast cancer research and treatment 2018, 167(2):417-423

46. Li VD, Li KH, Li JT: TP53 mutations as potential prognostic markers for specific cancers: analysis of data from The Cancer Genome Atlas and the International Agency for Research on Cancer TP53 Database. Journal of cancer research and clinical oncology 2019, 145(3):625-636.

47. Lacroix M, Riscal R, Arena G, Linares LK, Le Cam L: Metabolic functions of the tumor suppressor p53: Implications in normal physiology, metabolic disorders, and cancer. Molecular metabolism 2020, 33:2-22.

\section{Tables}

Table 1. Characteristics of all subjects in this study. 


\begin{tabular}{|c|c|c|c|c|c|}
\hline \multirow[t]{2}{*}{ Variables } & \multicolumn{2}{|c|}{ Verification phase $[\mathrm{N}=180[$} & \multicolumn{2}{|c|}{ Validation phase $(\mathrm{N}=252)$} & \multirow[t]{2}{*}{$P$} \\
\hline & $\operatorname{ESCC}(\mathrm{N}=90)$ & $\mathrm{NC}(\mathrm{N}=90)$ & $\operatorname{ESCC}(\mathrm{N}=126)$ & $\mathrm{NC}(\mathrm{N}=126)$ & \\
\hline \multicolumn{6}{|l|}{ Age } \\
\hline Median & 65 & 64 & 65 & 64 & $0.201^{\mathrm{a}}$ \\
\hline (Q1, Q3) & $(60.8,71.0)$ & $(57.0,68.3)$ & $(58.0,69.0)$ & $(57.0,69.0)$ & \\
\hline Range & $41-88$ & $47-84$ & $45-87$ & $43-83$ & $0.809^{b}$ \\
\hline \multicolumn{6}{|c|}{ GenderIn(\%) } \\
\hline Male & $60(66.7)$ & $60(66.7)$ & $86(68.3)$ & $86(68.3)$ & $0.643^{\mathrm{a}}$ \\
\hline Female & $30(31.3)$ & $30(33.3)$ & $40(31.7)$ & $40(31.7)$ & $0.806^{b}$ \\
\hline \multicolumn{6}{|c|}{ Family history[n(\%) } \\
\hline Yes & $15(16.7)$ & & $20 \rrbracket 15.9 \rrbracket$ & & $0.105^{\mathrm{a}}$ \\
\hline No & $74(82.2)$ & & $53 \llbracket 42.1 \rrbracket$ & & \\
\hline Unknown & $1(1.1)$ & & $53 \llbracket 42.1 \rrbracket$ & & \\
\hline \multicolumn{6}{|c|}{ Differentiated degreeln(\%) } \\
\hline Poorly & $13(14.4)$ & & 14(11.1) & & $0.837^{a}$ \\
\hline Moderately & $32(35.6)$ & & $32(25.4)$ & & \\
\hline Highly & $7(8.8)$ & & 14(11.1) & & \\
\hline unknown & $38(42.2)$ & & $66(52.4)$ & & \\
\hline \multicolumn{6}{|c|}{ TNM stage】n(\%) } \\
\hline 0 & $3(3.3)$ & & $8(6.3)$ & & $0.106^{a}$ \\
\hline$\square$ & 18(20.0) & & 25(19.8) & & \\
\hline ૧ & 14(15.6) & & $20(15.9)$ & & \\
\hline$\square$ & 18(20.0) & & 18(14.3) & & \\
\hline 口 & $9(10.0)$ & & $5(4.0)$ & & \\
\hline Unknown & 28(31.1) & & 50(39.7区 & & \\
\hline \multicolumn{6}{|c|}{ Degree of invasion $\square n(\%)$} \\
\hline T0-T1 & $22(23.7)$ & & $33 \rrbracket 26.2 \rrbracket$ & & $0.418^{\mathrm{a}}$ \\
\hline T2 & $8(8.6)$ & & $16 \rrbracket 12.7 \rrbracket$ & & \\
\hline Т3 & $31(33.3)$ & & $28 \llbracket 22.2 \rrbracket$ & & \\
\hline Unknown & $29(31.2)$ & & $49 \llbracket 38.9 \rrbracket$ & & \\
\hline
\end{tabular}

Note: a: there was no difference between subgroups.

b: there was no difference between verification phase and validation phase.

ESCC, esophageal squamous cell carcinoma; NC, normal control; Q1,25\% interquartile range; Q3,75\% interquartile range.

Table 2. Diagnostic value of 13 TAAbs in verification phase and validation phase for (ESCC) 


\begin{tabular}{|c|c|c|c|c|c|c|c|c|c|c|c|c|}
\hline \multirow[t]{2}{*}{ TAAbs } & \multicolumn{6}{|c|}{ Verification cohort (90 ESCC vs 90 NC) } & \multicolumn{6}{|c|}{ Validation cohort (126 ESCC vs 126 NC) } \\
\hline & $\mathrm{Se}(\%)$ & $\mathrm{Sp}(\%)$ & AUC & $95 \% \mathrm{Cl}$ & $\begin{array}{l}\text { Accuracy } \\
(\%)\end{array}$ & $P$ & $\mathrm{Se}(\%)$ & $\mathrm{Sp}(\%)$ & AUC & $95 \% \mathrm{Cl}$ & $\begin{array}{l}\text { Accuracy } \\
(\%)\end{array}$ & $P$ \\
\hline MAGEA1 & 33.3 & 91.1 & 0.670 & $\begin{array}{l}0.591- \\
0.749\end{array}$ & 62.2 & $<0.000$ & 40.5 & 90.1 & 0.743 & $\begin{array}{l}0.682- \\
0.803\end{array}$ & 65.3 & $<0.000$ \\
\hline NFKB1 & 35.6 & 91.1 & 0.675 & $\begin{array}{l}0.596- \\
0.754\end{array}$ & 63.3 & $<0.000$ & 32.5 & 90.9 & 0.626 & $\begin{array}{l}0.556- \\
0.695\end{array}$ & 61.7 & 0.001 \\
\hline VCL & 26.7 & 92.2 & 0.674 & $\begin{array}{l}0.597- \\
0.752\end{array}$ & 59.5 & $<0.000$ & 34.9 & 90.1 & 0.673 & $\begin{array}{l}0.607- \\
0.739\end{array}$ & 62.5 & $<0.000$ \\
\hline MAGEA4 & 25.6 & 91.1 & 0.609 & $\begin{array}{l}0.526- \\
0.692\end{array}$ & 58.3 & 0.012 & 37.3 & 90.1 & 0.640 & $\begin{array}{l}0.573- \\
0.708\end{array}$ & 63.7 & $<0.000$ \\
\hline PRKCZ & 26.7 & 92.2 & 0.648 & $\begin{array}{l}0.567- \\
0.728\end{array}$ & 59.5 & 0.001 & 36.5 & 90.6 & 0.674 & $\begin{array}{l}0.606- \\
0.741\end{array}$ & 63.6 & $<0.000$ \\
\hline TP53 & 17.8 & 98.9 & 0.648 & $\begin{array}{l}0.569- \\
0.728\end{array}$ & 58.3 & 0.001 & 39.7 & 91.5 & 0.695 & $\begin{array}{l}0.630- \\
0.761\end{array}$ & 65.6 & $<0.000$ \\
\hline ANKHD1 & 1.1 & 100.0 & 0.544 & $\begin{array}{l}0.460- \\
0.629\end{array}$ & 50.6 & 0.304 & - & - & - & - & - & - \\
\hline PPP1R15A & 16.7 & 90.0 & 0.569 & $\begin{array}{l}0.485- \\
0.653\end{array}$ & 53.3 & 0.108 & - & - & - & - & - & - \\
\hline SMARCC1 & 12.2 & 93.3 & 0.542 & $\begin{array}{l}0.458- \\
0.627\end{array}$ & 52.8 & 0.329 & - & - & - & - & - & - \\
\hline BASP1 & 4.4 & 96.7 & 0.507 & $\begin{array}{l}0.423- \\
0.591\end{array}$ & 50.6 & 0.880 & - & - & - & - & - & - \\
\hline GABPA & 15.6 & 90.0 & 0.508 & $\begin{array}{l}0.422- \\
0.593\end{array}$ & 52.8 & 0.856 & - & - & - & - & - & - \\
\hline SMARCE1 & 16.7 & 92.2 & 0.559 & $\begin{array}{l}0.475- \\
0.643\end{array}$ & 54.5 & 0.174 & - & - & - & - & - & - \\
\hline PDE4DIP & 17.8 & 90.0 & 0.537 & $\begin{array}{l}0.452- \\
0.622\end{array}$ & 53.9 & 0.396 & - & - & - & - & - & - \\
\hline
\end{tabular}

Note: ESCC, esophageal squamous cell carcinoma, NC, normal control, Se, sensitivity, Sp, specificity, AUC, area under the receiver operating characteristic curve, $\mathrm{Cl}$, confidence interval.

Table 3. Diagnostic value of 6 TAAbs for ESCC vs BED

\begin{tabular}{|llllll|}
\hline TAAbs & \multicolumn{7}{c|}{ 126 ESCC vs 237 BED } & & \\
& Se(\%) & Sp(\%) & AUC & $95 \%$ Cl & Accuracy (\%) \\
\hline MAGEA1 & 37.3 & 90.7 & 0.681 & $0.620-0.743$ & 72.2 \\
\hline NFKB1 & 32.5 & 91.1 & 0.651 & $0.589-0.713$ & 70.8 \\
\hline VCL & 38.9 & 90.3 & 0.704 & $0.646-0.761$ & 72.5 \\
\hline MAGEA4 & 43.6 & 90.7 & 0.764 & $0.712-0.816$ & 74.4 \\
\hline PRKCZ & 38.1 & 91.6 & 0.740 & $0.686-0.793$ & 73.0 \\
\hline TP53 & 37.3 & 92.4 & 0.700 & $0.642-0.758$ & 73.3 \\
\hline
\end{tabular}

Note: ESCC, esophageal squamous cell carcinoma, BED, benign esophageal disease, Se, sensitivity, Sp, specificity, AUC, area under the receiver operating characteristic curve; $\mathrm{Cl}$, confidence interval. 
Table 4. Diagnostic value of the TAAbs panel for ESCC patients with different subtypes

Page 12/19 


\begin{tabular}{|c|c|c|c|c|c|c|c|c|c|c|c|c|}
\hline & $\mathbf{N}$ & $\begin{array}{l}\mathrm{Se} \\
(\%)\end{array}$ & Sp(\%) & $\begin{array}{l}\text { Accuracy } \\
(\%)\end{array}$ & AUC & $95 \% \mathrm{Cl}$ & $P^{1}$ & $P^{2}$ & $\begin{array}{l}\text { PPV } \\
(\%)\end{array}$ & $\begin{array}{l}\text { NPV } \\
(\%)\end{array}$ & +LR & LR \\
\hline \multicolumn{13}{|l|}{$\begin{array}{l}\text { Verification } \\
\text { cohort }\end{array}$} \\
\hline All patients & 90 & 34.4 & 92.2 & 63.3 & 0.729 & $\begin{array}{l}0.656- \\
0.802\end{array}$ & & & 81.5 & 58.4 & 4.4 & 0.7 \\
\hline \multicolumn{13}{|l|}{ Age } \\
\hline$\leq 65$ & 48 & 22.9 & 94.4 & 69.5 & 0.681 & $\begin{array}{l}0.588- \\
0.774\end{array}$ & 0.422 & 0.109 & 68.6 & 69.7 & 4.1 & 0.8 \\
\hline$>65$ & 42 & 47.6 & 92.2 & 78.0 & 0.783 & $\begin{array}{l}0.698- \\
0.868\end{array}$ & 0.341 & & 74.0 & 79.0 & 6.1 & 0.6 \\
\hline \multicolumn{13}{|l|}{ Gender } \\
\hline Male & 60 & 35.5 & 92.2 & 69.1 & 0.721 & $\begin{array}{l}0.638- \\
0.804\end{array}$ & 0.886 & 0.705 & 75.8 & 67.5 & 4.6 & 0.7 \\
\hline Female & 30 & 35.7 & 91.1 & 78.0 & 0.746 & $\begin{array}{l}0.646- \\
0.845\end{array}$ & 0.787 & & 55.5 & 82.0 & 4.0 & 0.7 \\
\hline \multicolumn{13}{|c|}{ Family history } \\
\hline No & 74 & 35.1 & 92.2 & 66.4 & 0.727 & $\begin{array}{l}0.650- \\
0.805\end{array}$ & 0.971 & 0.899 & 78.7 & 63.3 & 4.5 & 0.7 \\
\hline Yes & 15 & 33.3 & 91.1 & 82.8 & 0.717 & $\begin{array}{l}0.583- \\
0.851\end{array}$ & 0.877 & & 38.4 & 89.1 & 3.7 & 0.7 \\
\hline \multicolumn{13}{|c|}{$\begin{array}{l}\text { Differentiated } \\
\text { degree }\end{array}$} \\
\hline Poorly & 13 & 38.5 & 95.6 & 88.4 & 0.756 & $\begin{array}{l}0.626- \\
0.887\end{array}$ & 0.721 & 0.510 & 55.8 & 91.5 & 8.7 & 0.6 \\
\hline $\begin{array}{l}\text { Highly and } \\
\text { moderately }\end{array}$ & 39 & 30.8 & 93.3 & 74.4 & 0.701 & $\begin{array}{l}0.601- \\
0.801\end{array}$ & 0.657 & & 66.6 & 75.7 & 4.6 & 0.7 \\
\hline \multicolumn{13}{|l|}{ TNM stage } \\
\hline 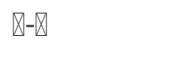 & 35 & 28.6 & 93.3 & 75.2 & 0.702 & $\begin{array}{l}0.603- \\
0.801\end{array}$ & 0.668 & 0.190 & 62.4 & 77.1 & 4.3 & 0.8 \\
\hline 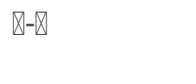 & 27 & 40.7 & 91.1 & 79.5 & 0.793 & $\begin{array}{l}0.700- \\
0.885\end{array}$ & 0.285 & & 57.8 & 83.7 & 4.6 & 0.7 \\
\hline \multicolumn{13}{|l|}{$\begin{array}{l}\text { Degree of } \\
\text { invasion }\end{array}$} \\
\hline T0-T1 & 24 & 25.0 & 94.4 & 79.8 & 0.650 & $\begin{array}{l}0.521- \\
0.780\end{array}$ & 0.296 & 0.130 & 54.3 & 82.5 & 4.5 & 0.8 \\
\hline T2-T3 & 39 & 38.5 & 91.1 & 75.2 & 0.770 & $\begin{array}{l}0.684- \\
0.855\end{array}$ & 0.476 & & 65.2 & 77.4 & 4.3 & 0.7 \\
\hline \multicolumn{13}{|c|}{ Validation cohort } \\
\hline All patients & 126 & 61.1 & 90.5 & 75.8 & 0.795 & $\begin{array}{l}0.739- \\
0.851\end{array}$ & & & 86.5 & 69.9 & 6.4 & 0.4 \\
\hline \multicolumn{13}{|l|}{ Age } \\
\hline & $\mathbf{N}$ & $\begin{array}{l}\mathrm{Se} \\
(\%)\end{array}$ & $\mathrm{Sp}(\%)$ & $\begin{array}{l}\text { Accuracy } \\
(\%)\end{array}$ & AUC & $95 \% \mathrm{Cl}$ & $P^{1}$ & $P^{2}$ & $\begin{array}{l}\text { PPV } \\
(\%)\end{array}$ & $\begin{array}{l}\text { NPV } \\
(\%)\end{array}$ & $+\mathrm{LR}$ & $\bar{L}$ \\
\hline$\leq 65$ & 68 & 63.2 & 90.5 & 80.9 & 0.784 & $\begin{array}{l}0.710- \\
0.858\end{array}$ & 0.818 & 0.651 & 78.2 & 82.0 & 6.7 & 0.4 \\
\hline$>65$ & 58 & 58.6 & 90.5 & 80.4 & 0.808 & $\begin{array}{l}0.736- \\
0.880\end{array}$ & 0.782 & & 74.0 & 82.6 & 6.2 & 0.5 \\
\hline
\end{tabular}




\begin{tabular}{|c|c|c|c|c|c|c|c|c|c|c|c|c|}
\hline \multicolumn{13}{|l|}{ Gender } \\
\hline Male & 83 & 55.4 & 90.1 & 76.3 & 0.768 & $\begin{array}{l}0.690- \\
0.836\end{array}$ & 0.553 & 0.127 & 78.7 & 75.4 & 5.6 & 0.5 \\
\hline Female & 43 & 72.1 & 90.5 & 85.8 & 0.848 & $\begin{array}{l}0.771- \\
0.925\end{array}$ & 0.276 & & 72.1 & 90.5 & 7.6 & 0.3 \\
\hline \multicolumn{13}{|c|}{ Family history } \\
\hline No & 53 & 56.6 & 90.5 & 80.5 & 0.805 & $\begin{array}{l}0.733- \\
0.878\end{array}$ & 0.832 & 0.237 & 71.5 & 83.2 & 6.0 & 0.5 \\
\hline Yes & 20 & 55.0 & 90.5 & 85.6 & 0.704 & $\begin{array}{l}0.554- \\
0.855\end{array}$ & 0.269 & & 47.9 & 92.7 & 5.8 & 0.5 \\
\hline \multicolumn{13}{|c|}{$\begin{array}{l}\text { Differentiated } \\
\text { degree }\end{array}$} \\
\hline Poorly & 14 & 64.3 & 90.5 & 87.9 & 0.763 & $\begin{array}{l}0.610- \\
0.916\end{array}$ & 0.701 & 0.688 & 42.9 & 95.8 & 6.8 & 0.4 \\
\hline $\begin{array}{l}\text { Highly and } \\
\text { moderately }\end{array}$ & 46 & 50.0 & 90.5 & 79.7 & 0.726 & $\begin{array}{l}0.631- \\
0.822\end{array}$ & 0.226 & & 65.8 & 83.2 & 5.3 & 0.6 \\
\hline \multicolumn{13}{|l|}{ TNM stage } \\
\hline 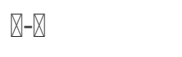 & 53 & 54.7 & 90.5 & 79.9 & 0.743 & \multirow{2}{*}{$\begin{array}{l}0.657- \\
0.830 \\
\\
0.643- \\
0.888\end{array}$} & 0.315 & \multirow[t]{2}{*}{0.731} & 70.8 & 82.6 & 5.8 & 0.5 \\
\hline 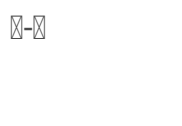 & 23 & 52.2 & 90.5 & 84.6 & 0.766 & & 0.715 & & 50.1 & 91.2 & 5.5 & 0.5 \\
\hline \multicolumn{13}{|l|}{$\begin{array}{l}\text { Degree of } \\
\text { invasion }\end{array}$} \\
\hline T0-T1 & 33 & 48.5 & 95.2 & 85.5 & 0.710 & $\begin{array}{l}0.596- \\
0.825\end{array}$ & 0.190 & 0.316 & 72.6 & 87.6 & 10.1 & 0.5 \\
\hline T2-T3 & 44 & 54.5 & 90.5 & 81.2 & 0.783 & $\begin{array}{l}0.697- \\
0.869\end{array}$ & 0.820 & & 66.7 & 85.1 & 5.7 & 0.5 \\
\hline
\end{tabular}

Note: ESCC, esophageal squamous cell carcinoma Se, sensitivity, Sp, specificity, AUC, area under the receiver operating characteristic curve; $C l$, confidence interval; $P P V$, positive predictive value; $N P V$, negative predictive value; $+L R$, positive likelihood ratio; $-L R$, negative likelihood ratio.

$P^{1}$ means comparison between TNM stage or family history or degree of invasion or distant metastasis or differentiated degree and all patients with the method of De Long et.

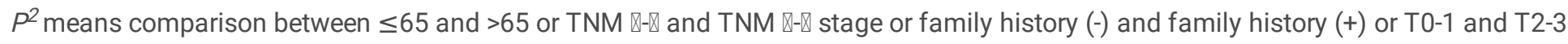
or poorly differentiated degree and highly and moderately differentiated degree.

\section{Figures}




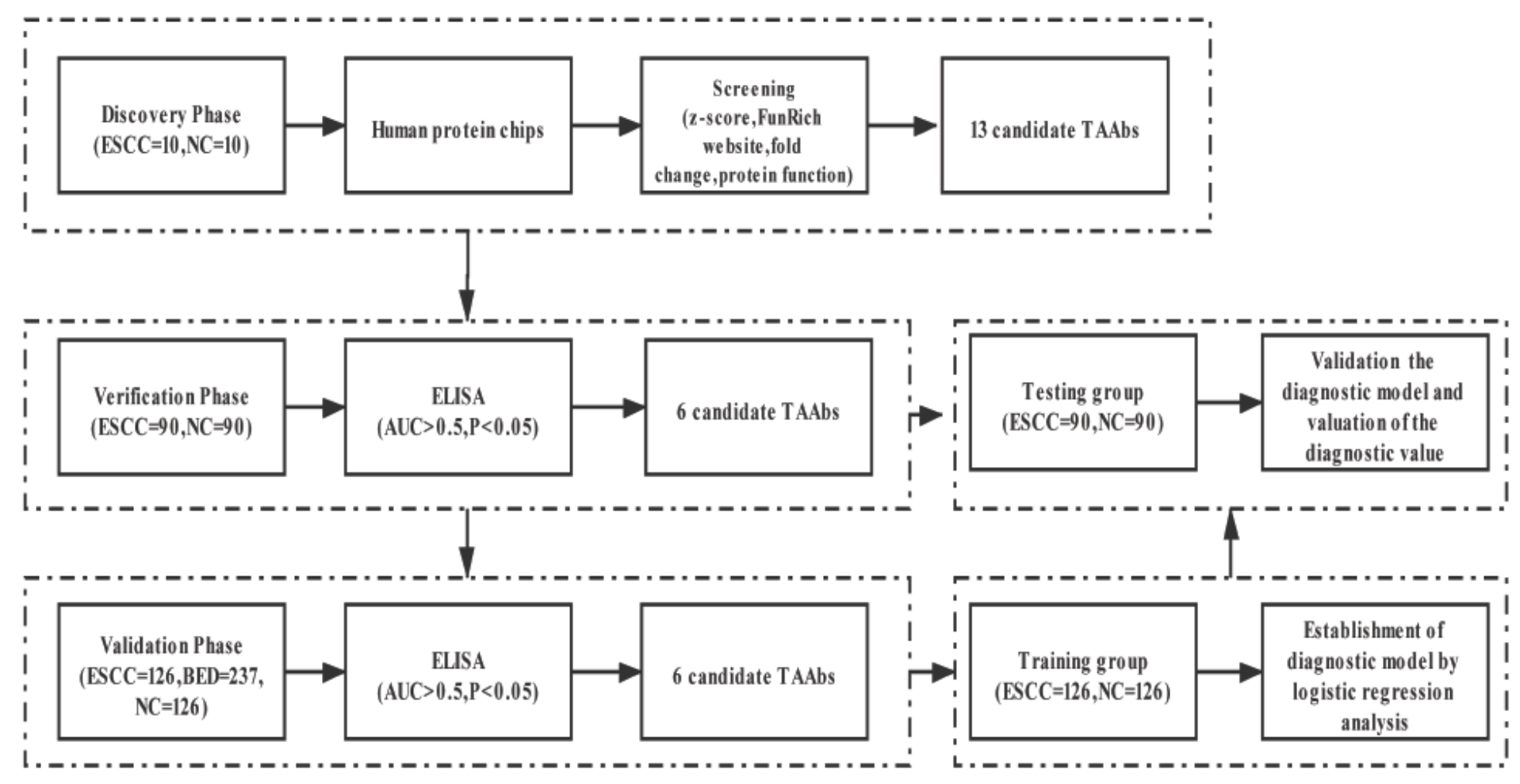

\section{Figure 1}

The design of this study. The first phase was the discovery phase, including 10 ESCC cases and $10 \mathrm{NCs}$, the high-throughput protein chips were used to screen candidate TAAbs related to the diagnosis of ESCC. The second phase was the verification phase, including 90 ESCC cases and 90 NCs, ELISA was used to verify the 13 TAAbs screened by the protein chips. The third phase was the validation phase, including 126 ESCC cases, 237 BED cases and 126 NCs. Furthermore, the candidate TAAbs screened out were validated by ELISA. ESCC: esophageal squamous cell carcinoma; NCs: normal controls; TAAbs: autoantibodies against tumor-associated antigens; ELISA: enzyme-linked immunosorbent assay. 

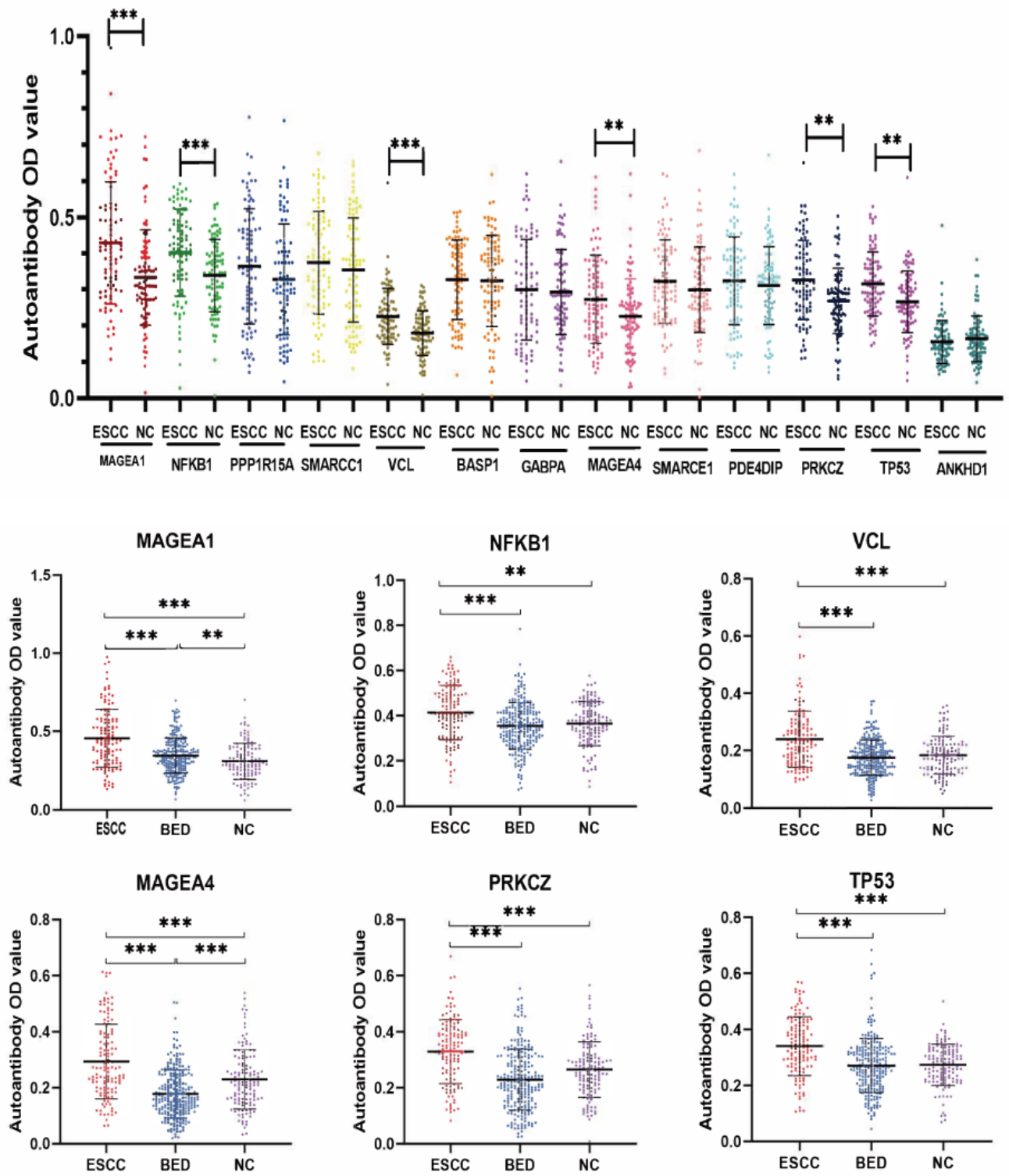

\section{Figure 2}

The titer of 13 TAAbs in ESCC cases and NCs. (A)Scatter plots of the titer of autoantibodies in the verification phase. (B)Scatter plots of the titer of autoantibodies in the validation phase. ESCC, esophageal squamous cell carcinoma; BED, benign esophageal diseases; NCs,

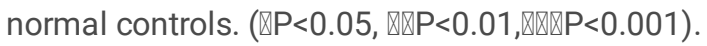



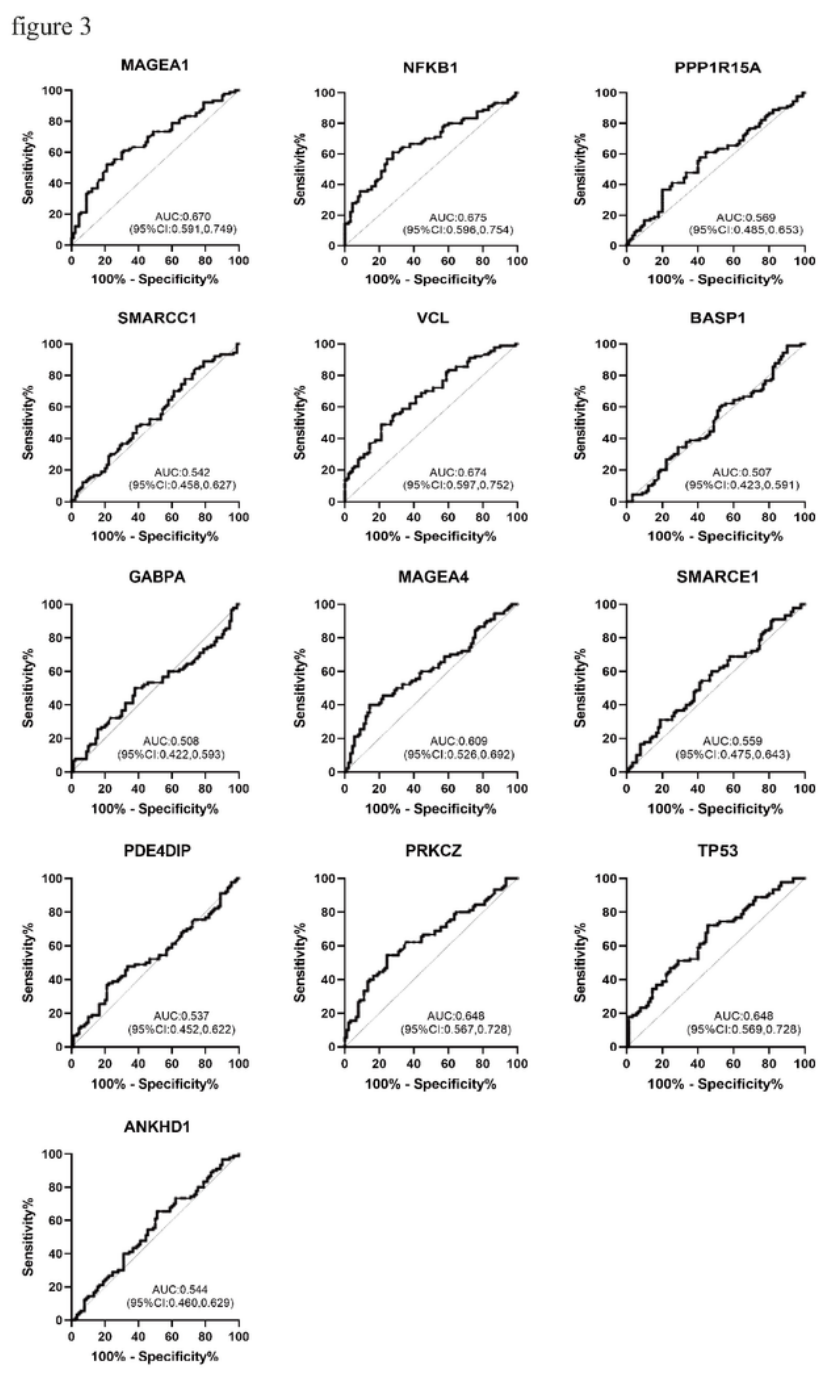

\section{Figure 3}

Diagnostic performance of 13 TAAbs in the verification phase for ESCC. ESCC, esophageal squamous cell carcinoma. 
MAGEA1:ESCC vs NC

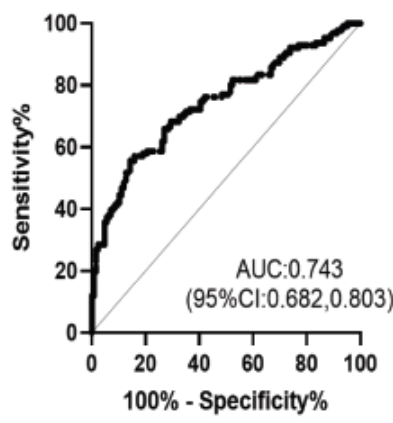

VCL:ESCC vs NC

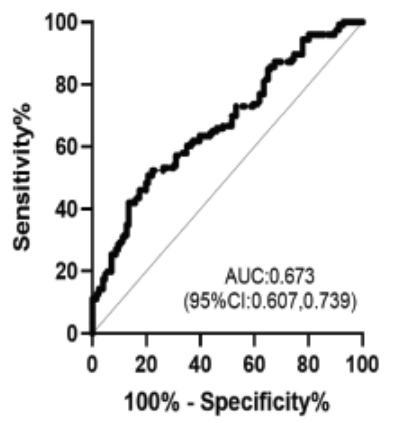

PRKCZ:ESCC vs NC

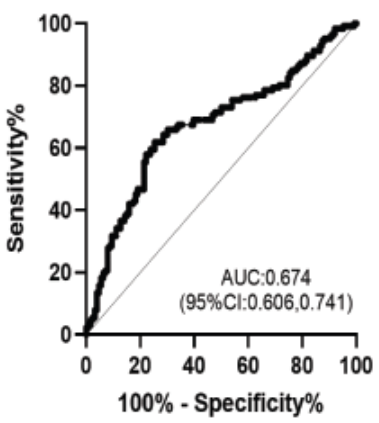

MAGEA1:ESCC vs BED

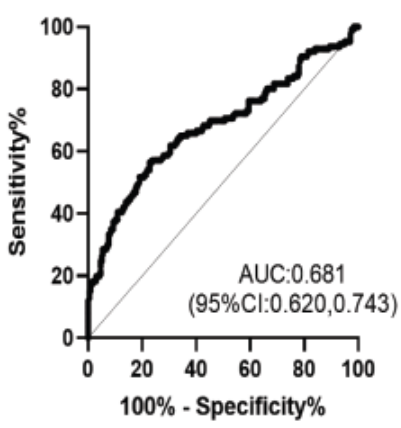

VCL:ESCC vs BED

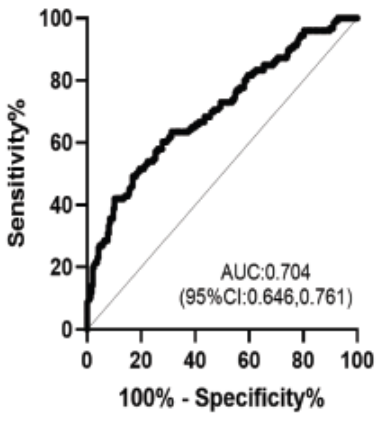

PRKCZ:ESCC vs BED

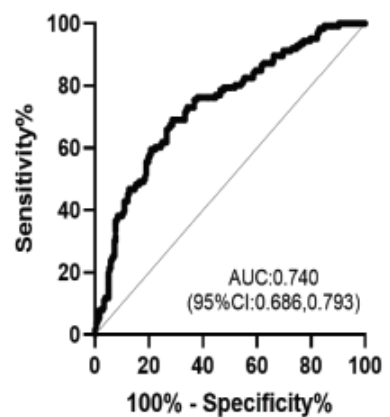

NFKB1:ESCC vs NC

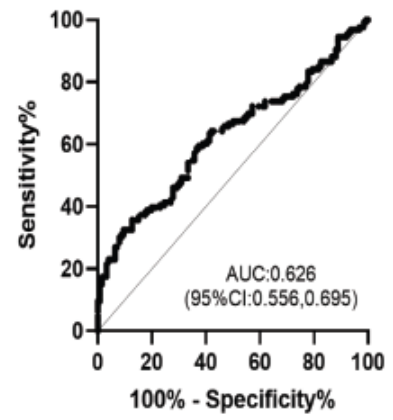

MAGEA4:ESCC vs NC

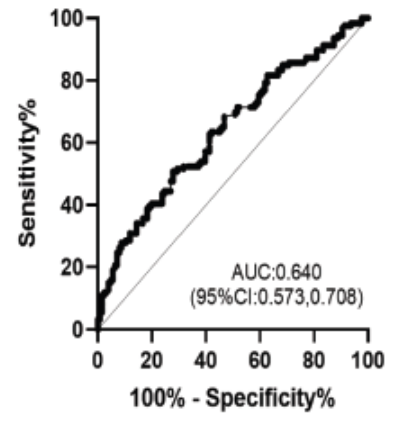

TP53:ESCC vs NC

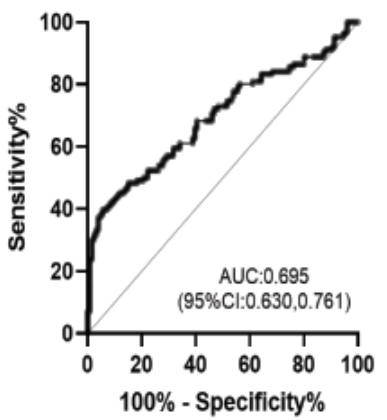

NFKB1: ESCC vs BED

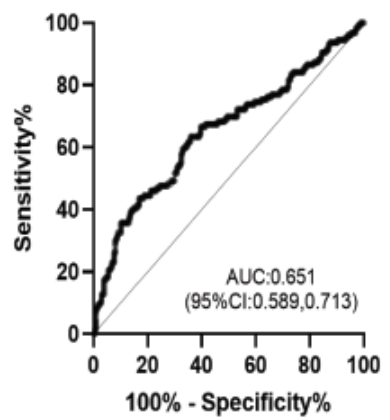

MAGEA4:ESCC vs BED

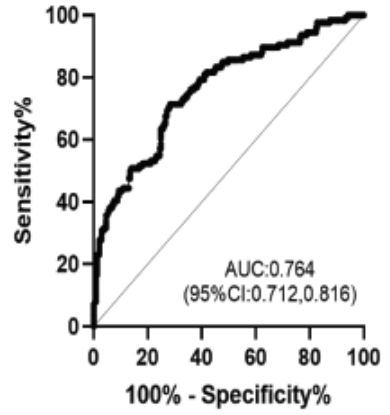

TP53:ESCC vs BED

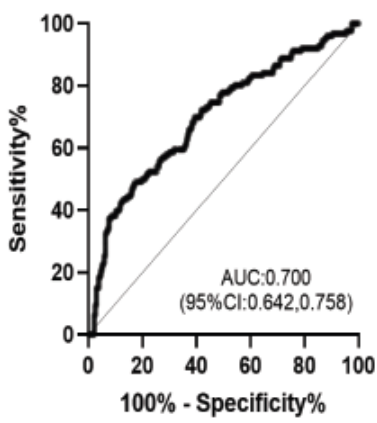

Figure 4

Diagnostic performance of 6 TAAbs in the validation phase for ESCC and BED detection. ESCC, esophageal squamous cell carcinoma, BED, benign esophageal diseases, NC, normal control. 
A

3TAAbs in Training group

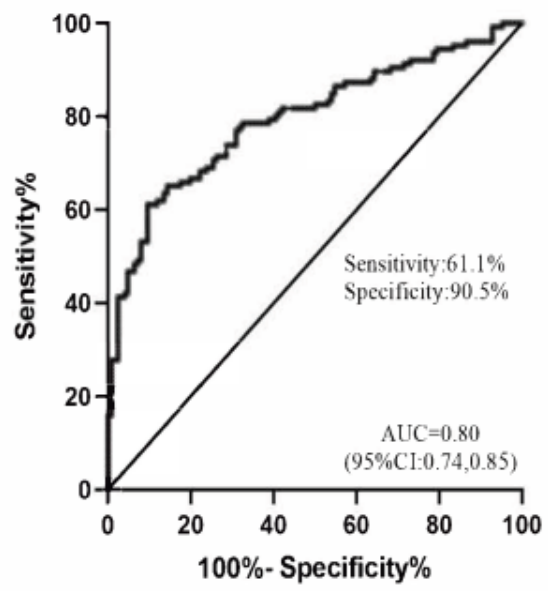

3TAAbs in Testing group

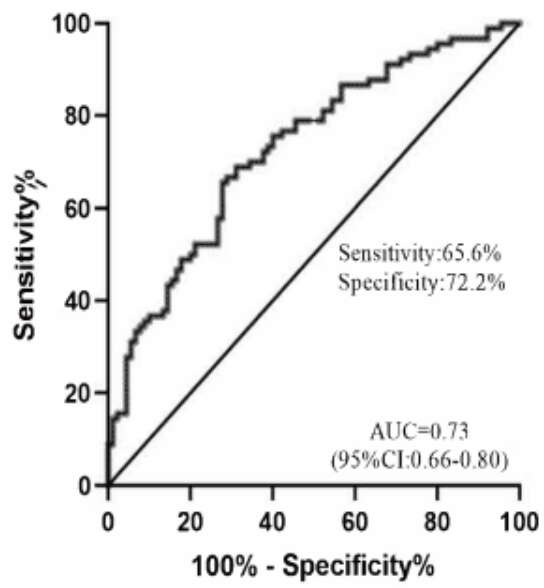

3TAAbs in BED group

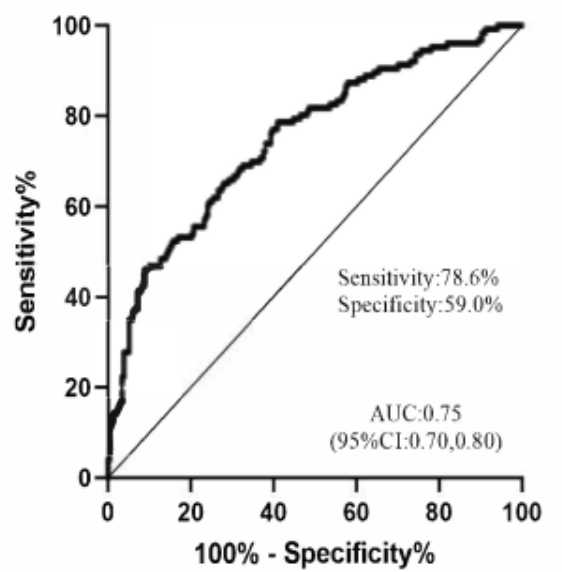

Figure 5

Receiver operating characteristic curve analysis of the prediction model with TAAbs panel in esophageal squamous cell carcinoma (ESCC) detection. (A)The prediction model with 3TAAbs (anti-MAGEA1, anti-VCL, anti-TP53) in the training group. (B) The prediction model with 3TAAbs (anti-MAGEA1, anti-VCL, anti-TP53) in the testing group. (C)The diagnosis performance of the prediction model for benign esophageal diseases (BED) cases.

\section{Supplementary Files}

This is a list of supplementary files associated with this preprint. Click to download.

- figures1.pdf

- figures2.pdf

- figureS3.pdf 